\title{
Chiral symmetry in the 2-flavour lattice Schwinger model*
}

\author{
I. Hip ${ }^{\mathrm{a}}$, C. B. Lang ${ }^{\mathrm{a}}$ and $\quad$ R. Teppner ${ }^{\mathrm{a}}$ \\ ${ }^{a}$ Institut für Theoretische Physik, Universität Graz, A-8010 Graz, Austria
}

We study the 2-flavour lattice Schwinger model: QED in $D=2$ with two fermion species of identical mass. In the simulation we are using Wilson fermions where chiral symmetry is explicitly broken. Since there is no known simple order parameter it is non-trivial to identify the critical line of the chiral phase transition. We therefore need to find observables which allow an identification of a possible restoration of chiral symmetry. We utilize the PCAC-relations in order to identify the critical coupling, where chiral symmetry is restored.

\section{INTRODUCTION}

The 2-flavour Schwinger model is a 2D theory of two fermion species and photons. In the massless continuum model the $S U(2)_{A}$ flavour symmetry is broken by a mechanism mimicking spontaneous symmetry breaking. Due to the MerminWagner-Coleman theorem [1] in this situation there is no spontaneous symmetry breaking and thus there are no Goldstone bosons, nevertheless massless pions are expected from continuum studies (for a recent study cf. [2]).

The naive lattice formulation for fermions shows the notorious doubling problem. In Wilson's action a term is added giving the doublers a mass $\mathcal{O}(1 / a)$ decoupling them in the continuum limit. Independent of the possible spontaneous breaking of chiral symmetry (which does not occur in $2 \mathrm{D}$ but is expected in e.g. $\mathrm{QCD}_{4}$ ) we expect that the chiral symmetry of the action itself is restored at a critical fermion coupling $\kappa_{c}(\beta)$. However, since the Wilson term breaks chiral symmetry explicitly we have no known simple order parameter, which allows one to identify that position. Following earlier proposals [3] it has recently been suggested to utilize PCACrelations with Schrödinger functional methods for that purpose [4]; this turned out to be quite powerful in the context of $\mathrm{QCD}_{4}$ [5]. Here we report on results for $\mathrm{QED}_{2}$ with 2 species of fermions, where we also employed PCAC-relations in order

\footnotetext{
*Supported by Fonds zur Förderung der Wissenschaftlichen Forschung in Österreich, Project P11502-PHY.

${ }^{\ddagger}$ presented by R. Teppner
}

to determine the critical fermion coupling line.

The lattice action for the massive 2-flavour Schwinger model with Wilson fermions is

$S[U, \psi, \bar{\psi}]=S_{g}[U]+S_{f}[U, \psi, \bar{\psi}]$,

where $S_{g}$ is the compact plaquette gauge action (coupling $\beta), \psi=(u, d)$ is a flavour-doublet and

$$
\begin{aligned}
& S_{f}[U, \psi, \bar{\psi}]=\sum_{n} \bar{\psi}_{n} \psi_{n} \\
& \quad-\kappa \sum_{n, \mu}\left[\bar{\psi}_{n}\left(r-\sigma^{\mu}\right) U_{n, \mu} \psi_{n+\mu}+h . c .\right] .
\end{aligned}
$$

The $\bar{\psi} \psi$ term and the terms proportional to $r$ (we choose $r=1$ ) break chiral symmetry explicitly. Strictly speaking the action has only the $S U(2)_{V} \times U(1)_{V}$ global symmetries. The main idea using the PCAC-concept is to find the points in the coupling space, where the explicit breaking of the chiral symmetries $S U(2)_{A} \times U(1)_{A}$ vanishes. The fermions were simulated with the HMC-method; more details can be found in 66.

\section{WARD IDENTITIES}

Global symmetries of an action on the classical level lead to conserved Noether-currents. In a QFT the symmetries may be spontaneously broken or broken due to anomalies resulting from the functional integration. So the relations expressing current conservation may change depending on the mechanism of symmetry violation. To derive these relations one considers global infinitesimal symmetry transformations of a path integral

$\langle 0|\mathcal{O}[U, \bar{\psi}, \psi]| 0\rangle=\frac{1}{Z} \int d U d \bar{\psi} d \psi \mathcal{O}[U, \bar{\psi}, \psi] e^{-S},(3)$ 


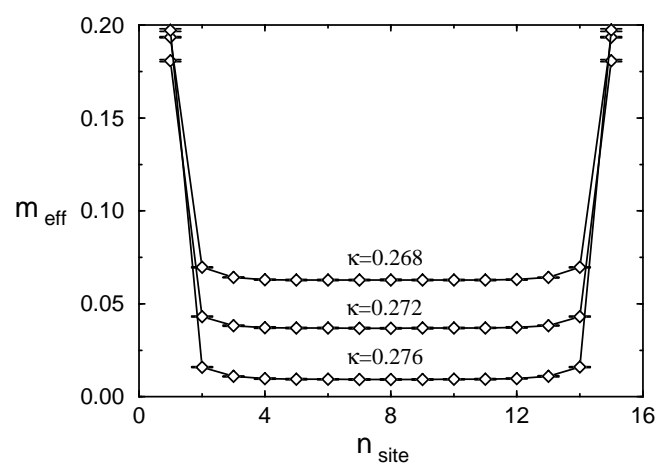

Figure 1. Mass plateaus at $\beta=2.0$ for the $16 \times 16$ lattice (10000 configurations).

where $\mathcal{O}$ denotes an arbitrary operator. If the integration measure is invariant this leads to relations like

$\langle 0|\delta \mathcal{O}| 0\rangle-\langle 0|\mathcal{O} \delta S| 0\rangle=0$,

called Ward identities. Thus, the quantum analog to the classical conservation law has similar (or equal) form but is an operator identity.

For the massive continuum Schwinger model with two flavours the corresponding identities for the global $S U(2)_{V} \times U(1)_{V}$ symmetries which are exactly realized in the QFT are

- $S U(2)_{V} \quad \partial_{\mu} j^{\mu a}=0, j^{\mu a}=\bar{\psi} \frac{\tau^{a} \otimes \sigma^{\mu}}{2} \psi,(5)$

- $U(1)_{V} \quad \partial_{\mu} j^{\mu}=0, j^{\mu}=\bar{\psi} \frac{1_{2 \times 2} \otimes \sigma^{\mu}}{2} \psi$.(6)

The $S U(2)_{A} \times U(1)_{A}$ symmetries are broken for massive fermions. The $S U(2)_{A}$ Ward identity (PCAC-relation) reads

- $S U(2)_{A} \quad \partial_{\mu} j^{\mu a 3}=2 m_{0} \pi^{a}, \pi^{a}=\bar{\psi} \frac{\tau^{a} \otimes \sigma^{3}}{2} \psi$

$$
j^{\mu a 3}=\bar{\psi} \frac{\tau^{a} \otimes \sigma^{\mu} \sigma^{3}}{2} \psi .
$$

The $U(1)_{A}$ symmetry is also broken by the anomaly

- $U(1)_{A} \quad \partial_{\mu} j^{\mu 3}=2 m_{0} \bar{\psi} \frac{1_{2 \times 2} \otimes \sigma^{3}}{2} \psi+$ anomaly, $j^{\mu 3}=\bar{\psi} \frac{1_{2 \times 2} \otimes \sigma^{\mu} \sigma^{3}}{2} \psi$.

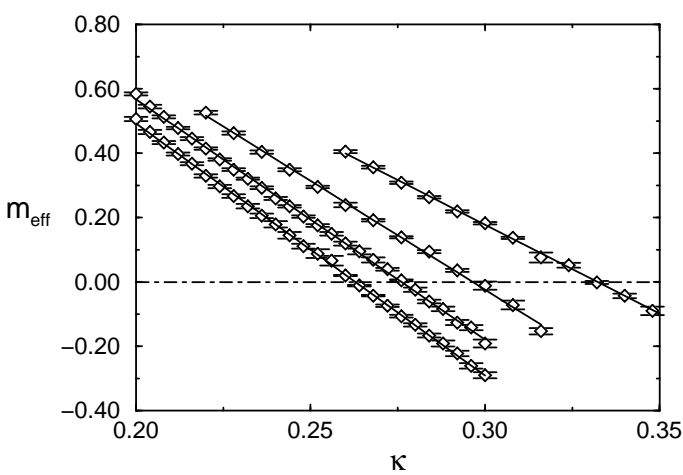

Figure 2. Values (and linear fits) obtained for $\hat{m}_{\text {eff }}$ for different $\kappa$ at a given $\beta$ (from left to right: $0.1,1,2,4)$ on an $8 \times 8$ lattice (1000 configurations each).

\section{RESULTS}

The underlying concept is to utilize a lattice version of $(7)$ in order to identify the value of $\kappa_{c}(\beta)$ where $S U(2)_{A}$ holds. That point defines a theory with massless lattice fermions. The naively discretized continuum action

$$
\begin{aligned}
& S=S_{g}[U]+\hat{m}_{\mathrm{eff}} \sum_{n} \bar{\psi}_{n} \psi_{n} \\
& -\frac{1}{2} \sum_{n \mu}\left\{\bar{\psi}_{n+\mu} \sigma^{\mu} U_{n, \mu}^{+} \psi_{n}+\bar{\psi}_{n}\left(-\sigma^{\mu}\right) U_{n, \mu} \psi_{n+\mu}\right\}
\end{aligned}
$$

leads to an $S U(2)_{A}$ lattice operator identity analogous to (7). In order to satisfy that relation non-trivially we choose to take expectation values with regard to a pion source and obtain

$$
\begin{aligned}
& \forall n \neq y: \quad 2 \hat{m}_{\mathrm{eff}}\left\langle 0\left|\pi_{y}^{a+} \pi_{n}^{a}\right| 0\right\rangle= \\
& \quad \sum_{\mu}\left[\left\langle 0\left|\pi_{y}^{a+} \hat{J}_{n}^{3 a \mu}\right| 0\right\rangle-\left\langle 0\left|\pi_{y}^{a+} \hat{J}_{n-\mu}^{3 a \mu}\right| 0\right\rangle\right]
\end{aligned}
$$

with

$$
\begin{array}{r}
\hat{J}_{n}^{3 a \mu}=\frac{1}{2}\left[\bar{\psi}_{n+\mu} U_{n, \mu}^{+} \frac{\tau^{a} \otimes \sigma^{\mu} \sigma^{3}}{2} \psi_{n}+\right. \\
\left.\bar{\psi}_{n} \frac{\tau^{a} \otimes \sigma^{\mu} \sigma^{3}}{2} U_{n, \mu} \psi_{n+\mu}\right] .
\end{array}
$$




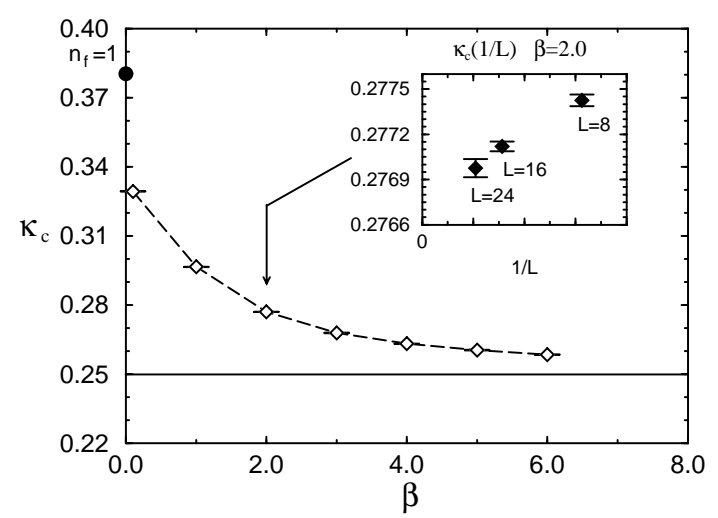

Figure 3. Phase diagram for the 2-flavour model for the $16 \times 16$ lattice (dashed lines to guide the eye); the value for the 1 -flavour model is from [7].

The operators in the above relation are projected onto states with zero momentum by summing over time-slices. This relation enables us to measure the mass parameter $\hat{m}_{\text {eff }}$. Here we are only interested in the value $\kappa_{c}(\beta)$, where $\hat{m}_{\text {eff }}$ vanishes.

The identity (10) should hold independent of the time-separation between source and sink. In [4] such a behaviour in the context of the Schrödinger functional is used to determine the value of improvement coefficients. In our case we find plateaus already as soon as the distance extends beyond the overlap region of the operators (fig. 1). For the analysis we excluded the points up to distance 3 (for $8 \times 8$ lattice: 2 ).

The remaining points represent a mass plateau giving the value of $\hat{m}_{\mathrm{eff}}$ and its error is obtained from the weighted average. The errors of the correlation functions were computed using the jackknife method.

For given $\beta$ we determined the values of $\hat{m}_{\text {eff }}$ for different values of $\kappa$. According to the general idea chiral symmetry is restored where $\hat{m}_{\text {eff }}$ vanishes. Interpolating the values of $\hat{m}_{\mathrm{eff}}$ we get the desired value of $\kappa_{c}(\beta)$ (fig. 2). In the domain studied a linear fit is appropriate. The resulting phase diagram is given in fig. 3 .

Finally, fig. 1 indicates possible problems. When measuring $\hat{m}_{\text {eff }}$ we simultaneously kept track of the topological charge $\nu$ of the configura-

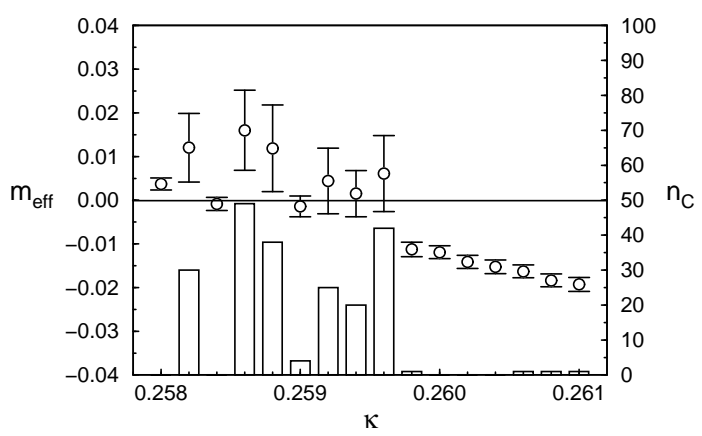

Figure 4. Dependence of $\hat{m}_{\text {eff }}$ (circles) on the topological charge $\nu(\beta=6$, lattice size $8 \times 8$, 1000 configurations each; $n_{c}$ denotes the number of configurations with non-zero $\nu$ ).

tions. In particular at larger $\beta$ tunneling between different sectors becomes less frequent [8]. Our results demonstrate a distinct dependence of the measured $\hat{m}_{\text {eff }}$ on $\nu$ which may lead to problems in situations where a simulation is non-ergodic.

\section{REFERENCES}

1. N. D. Mermin and H. Wagner, Phys. Rev. Lett. 17 (1966) 1133; S. Coleman, Commun. Math. Phys. 31 (1973) 259.

2. C. R. Gattringer and E. Seiler, Ann. Phys. 233 (1994) 97.

3. M. Bochicchio, L. Maiani, G. Martinelli, G. Rossi and M. Testa, Nucl. Phys. B262 (1985) 331.

4. K. Jansen, C. Liu, M. Lüscher, H. Simma, S. Sint, R. Sommer, P.Weisz and U. Wolff, Phys.Lett. B372 (1996) 275.

5. M. Lüscher, talk at this conference.

6. C.R. Gattringer, I. Hip and C.B. Lang, preprints hep-lat/9706010 (to appear in Phys. Lett.), hep-lat/9707011 and contrib. to this conference.

7. H. Gausterer and C. B. Lang, Nucl. Phys. B455 (1995) 785.

8. A. van der Sijs, contrib. to this conference. 